PROCEEDINGS OF THE

AMERICAN MATHEMATICAL SOCIETY

Volume 138, Number 4, April 2010, Pages 1433-1438

S 0002-9939(09)10176-4

Article electronically published on December 8, 2009

\title{
HOMOMORPHISM OF QUASIANALYTIC LOCAL RINGS
}

\author{
ABDELHAFED ELKHADIRI
}

(Communicated by Ted Chinburg)

\begin{abstract}
Let $\mathcal{C}_{n}$ be a local quasi-analytic subring of the ring of germs of $C^{\infty}$ functions on $\mathbb{R}^{n}$, and let $\mathcal{C}=\left\{\mathcal{C}_{n}, n \in \mathbb{N}\right\}$. We suppose that $\mathcal{C}$ is closed under composition. Consider a map $\varphi:\left(\mathbb{R}^{n}, 0\right) \rightarrow\left(\mathbb{R}^{k}, 0\right)$ vanishing at zero, where $\varphi$ is a $k$-tuple $\left(\varphi_{1}, \ldots, \varphi_{k}\right)$ and $\varphi_{1}, \ldots, \varphi_{k}$ are in $\mathcal{C}_{n}$. Then $\varphi$ defines uniquely a map $\phi: \mathcal{C}_{k} \rightarrow \mathcal{C}_{n}$ by composition, and $\phi$ induces a morphism $\hat{\phi}: \hat{\mathcal{C}_{k}} \rightarrow \hat{\mathcal{C}_{n}}$ between completions. We let $\phi_{*}: \frac{\hat{\mathcal{C}_{k}}}{\mathcal{C}_{k}} \rightarrow \hat{\widehat{\mathcal{C}_{n}}}$ be the homomorphism of groups induced by $\phi$ and $\hat{\phi}$ in the obvious manner. In the analytic case, i.e. when each $\mathcal{C}_{n}$ is the ring of germs of real analytic functions, M. Eakin and A. Harris give a condition under which $\phi_{*}$ is injective. In this paper we prove that the same statement does not hold for a quasianalytic system unless this system is analytic.
\end{abstract}

\section{INTRODUCTION}

Given local rings $A$ and $B$ and a local homomorphism $\phi: A \rightarrow B$, let $\hat{\phi}$ : $\hat{A} \rightarrow \hat{B}$ be the induced morphism between completions. Let $\phi_{*}: \frac{\hat{A}}{A} \rightarrow \frac{\hat{B}}{B}$ be the homomorphism of abelian groups induced by $\phi$ and $\hat{\phi}$ in the obvious manner. We say that $\phi$ is strongly injective if $\phi_{*}$ is injective. Suppose now that $A$ and $B$ are the rings of convergent power series; i.e. $A=\mathbb{R}\left\{x_{1}, \ldots, x_{n}\right\}$ and $B=\mathbb{R}\left\{y_{1}, \ldots, y_{k}\right\}$. Suppose $\varphi=\left(\varphi_{1}, \ldots, \varphi_{k}\right), \varphi_{j} \in \mathbb{R}\left\{x_{1}, \ldots, x_{n}\right\}, j=1, \ldots, k$, and $\varphi_{1}(0)=\ldots=\varphi_{k}(0)=0$.

Let $\phi: \mathbb{R}\left\{y_{1}, \ldots, y_{k}\right\} \rightarrow \mathbb{R}\left\{x_{1}, \ldots, x_{n}\right\}$ be the morphism defined by composition. Let $r k(\varphi)$ denote the rank of the jacobian matrix $\left[\frac{\partial \varphi_{j}}{\partial x_{i}}\right]$, considered as the matrix over the quotient field of $\mathbb{R}\left\{x_{1}, \ldots, x_{n}\right\}$. M. Eakin and A. Harris proved that $\phi$ is strongly injective if and only if $r k(\varphi)=k$ 2. Their result extends a result of Abhyankar and Van der Put 1]. In this paper we prove that if the result of M. Eakin and A. Harris is true for quasianalytic local rings, then these rings are analytic. See Corollary 4.1 below.

Definition 1.1. A differentiable system is a sequence $\mathcal{C}=\left\{\mathcal{C}_{n}, n \in \mathbb{N}\right\}$ such that, for each $n \in \mathbb{N}, \mathcal{C}_{n} \subset \mathcal{E}_{n}$ is a local subring of the ring of germs, at the origin of $\mathbb{R}^{n}$, of $C^{\infty}$ functions. We suppose that, for each $n \in \mathbb{N}, \mathcal{C}_{n}$ is closed upon taking derivatives and the following holds:

Received by the editors May 19, 2008, and, in revised form, August 23, 2009, and August 25, 2009 .

2010 Mathematics Subject Classification. Primary 26E10, 32B05; Secondary 58C10.

Key words and phrases. Weierstrass division theorem, quasianalytic local rings.

This work was partially supported by PARS MI 33.

(C)2009 American Mathematical Society Reverts to public domain 28 years from publication 
$\left(C_{1}\right) \mathbb{R}\left[x_{1}, \ldots, x_{n}\right] \subset \mathcal{C}_{n} \subset \mathcal{E}_{n}$, for each $n \in \mathbb{N}$, where $\mathbb{R}\left[x_{1}, \ldots, x_{n}\right]$ is the ring of polynomials with coefficients in $\mathbb{R}$.

$\left(C_{2}\right)$ The system $\mathcal{C}$ is closed under composition. This means that if $g \in \mathcal{C}_{k}$ and $f=\left(f_{1}, \ldots, f_{k}\right) \in\left(\mathcal{C}_{n}\right)^{k}$ with $f(0)=0$, then $g \circ f \in \mathcal{C}_{n}$.

$\left(C_{3}\right)$ For each $n \in \mathbb{N}, \mathcal{C}_{n}$ is closed under division by coordinates. This means that if $f \in \mathcal{C}_{n}$ and $f=\left(x_{i}-\alpha\right) g$, where $g \in \mathcal{E}_{n}$, and $\alpha \in \mathbb{R}$, then $g \in \mathcal{C}_{n}$.

$\left(C_{4}\right)$ The Implicit Function Theorem for $\mathcal{C}_{n}$ holds in the following sense: Suppose that $f=\left(f_{1}, \ldots, f_{m}\right) \in\left(\mathcal{C}_{n+m}\right)^{m}$ with $f(0,0)=0$. Put $y=\left(y_{1}, \ldots, y_{m}\right)$ and suppose that

$$
\operatorname{det}\left(\frac{\partial f_{i}}{\partial y_{j}}(0,0)\right)_{i, j=1 \ldots m} \neq 0 .
$$

Then there is a (unique ) $g=\left(g_{1}, \ldots, g_{m}\right) \in\left(\mathcal{C}_{n}\right)^{m}$ with $g(0)=0$ such that $f(x, g(x))=0$.

Call

$$
\therefore: \mathcal{C}_{n} \rightarrow \mathbb{R}\left[\left[x_{1}, \ldots, x_{n}\right]\right]
$$

the map which associates to each $f \in \mathcal{C}_{n}$ its Taylor expansion at the origin. We consider the following condition:

$\left(C_{5}\right) \hat{\imath}$ is an injective homomorphism.

Definition 1.2. A differentiable system is called quasianalytic if condition $\left(C_{5}\right)$ holds.

In the following, for a differentiable quasianalytic system we will not distinguish notationally between the germ and its image by ., i.e. its Taylor expansion at the origin.

We say that the Weierstrass Division Theorem holds in the differentiable quasianalytic system $\left(\mathcal{C}_{n}\right)_{n}$ if the following condition, $\left(W_{n}\right)$, is satisfied for each $n \in \mathbb{N}$ :

If $f \in \mathcal{C}_{n}$ and $f\left(0, X_{n}\right) \in \mathbb{R}\left[\left[X_{n}\right]\right]$ is nonzero of order $d$, then for every $g \in \mathcal{C}_{n}$ there is a $q \in \mathcal{C}_{n}$ and there are $r_{i} \in \mathcal{C}_{n-1}, i=0, \ldots, d-1$, such that

$$
g=q f+\left(r_{d-1} X_{n}^{d-1}+\ldots+r_{0}\right) .
$$

We will use the following theorem, proved in [4], where $\mathbb{R}\left\{x_{1}, \ldots, x_{n}\right\}$ is the ring of convergent power series.

Theorem 1.3. Assume that the property $\left(W_{n}\right)$ holds, for each $n \in \mathbb{N}$, in the differentiable quasianalytic system $\left(\mathcal{C}_{n}\right)_{n \in \mathbb{N}}$. Then for every $n \in \mathbb{N}$, we have $\mathcal{C}_{n} \subseteq$ $\mathbb{R}\left\{x_{1}, \ldots, x_{n}\right\}$.

Throughout this paper, let $\mathcal{C}=\left\{\mathcal{C}_{n}, n \in \mathbb{N}\right\}$ denote a fixed (but arbitrary) differentiable quasianalytic system.

Let $\varphi=\left(\varphi_{1}, \ldots, \varphi_{k}\right)$ with $\varphi_{i} \in \mathcal{C}_{n}, i=1, \ldots, k$ and $\varphi(0)=0$, and let $x=$ $\left(x_{1}, \ldots, x_{n}\right)$ and $y=\left(y_{1}, \ldots, y_{k}\right)$ be the coordinates in $\mathbb{R}^{n}$ and $\mathbb{R}^{k}$ respectively. The generic rank of $\varphi, r k(\varphi)$, is the rank of the Jacobian matrix $\left[\frac{\partial \varphi_{i}}{\partial x_{j}}\right]$, considered as a matrix over the quotient field of $\mathcal{C}_{n}$ (recall that $\mathcal{C}_{n}$ is a domain by $\left(C_{5}\right)$ ). We consider the morphism $\phi: \mathcal{C}_{k} \rightarrow \mathcal{C}_{n}$ defined by composition by $\varphi$ and its extension to the completion $\hat{\phi}: R\left[\left[y_{1}, \ldots, y_{k}\right]\right] \rightarrow R\left[\left[x_{1}, \ldots, x_{n}\right]\right]$.

Definition 1.4. We say that the differentiable quasianalytic system $\mathcal{C}$ has the property of M. Eakin and A. Harris (EH, for short) [2] if for each $\varphi$ as above with 
$r k(\varphi)=k$ and for each $\psi \in \mathbb{R}\left[\left[y_{1}, \ldots, y_{k}\right]\right]$ such that $\hat{\phi}(\psi)=\hat{g}$, with $g \in \mathcal{C}_{n}$, there exists $\beta \in \mathcal{C}_{k}$ such that $\hat{\beta}=\psi$.

\section{Quasianalytic version of the Newton Theorem}

If $\lambda=\left(\lambda_{1}, \ldots, \lambda_{p}\right) \in \mathbb{R}^{p}$, then $P\left(x_{n}, \lambda\right)=x_{n}^{p}+\sum_{i=1}^{p} \lambda_{i} x_{n}^{p-i}$ is called a generic polynomial in $x_{n}$ of degree $p$.

Let $\sigma=\left(\sigma_{1}, \ldots, \sigma_{p}\right): \mathbb{R}^{p} \rightarrow \mathbb{R}^{p}$ be the polynomial map defined by $\sigma_{i}=(-1)^{-i} \tilde{\sigma}_{i}$, where the $\tilde{\sigma}_{i}$ are the elementary symmetric functions in the variables $\lambda_{1}, \ldots, \lambda_{p}$. Then we have $P\left(x_{n}, \sigma(\lambda)\right)=\left(x_{n}-\lambda_{1}\right) \ldots\left(x_{n}-\lambda_{p}\right)$.

The function $\varphi(x, \lambda)=(x, \sigma(\lambda))$ defines, by substitution, a morphism $\phi: \mathcal{C}_{n+p} \rightarrow$ $\mathcal{C}_{n+p}$. We can see that $r k(\varphi)=n+p$ and $\phi$ is an injective morphism.

Lemma 2.1. Suppose that the system $\mathcal{C}$ has the property $E H$ and let $f(x, \lambda) \in \mathcal{C}_{n+p}$ be a function germ symmetric with respect to the variables $\lambda_{i}, i=1, \ldots, p$. Then there exists $g \in \mathcal{C}_{n+p}$ such that $g(x, \sigma(\lambda))=f(x, \lambda)$.

Proof. By hypothesis $\hat{f}(x, \lambda) \in \mathbb{R}[[x, \lambda]]$ is symmetric with respect to the variables $\lambda_{i}, i=1, \ldots, p$. By the Newton Theorem (for formal series) there exists $h \in \mathbb{R}[[x, \lambda]]$ such that $h(x, \sigma(\lambda))=\hat{f}(x, \lambda)$; i.e. $\hat{\phi}(h)=\hat{f}$. Since $r k(\varphi)=n+p[\varphi(x, \lambda)=$ $(x, \sigma(\lambda))]$ and the system $\mathcal{C}$ is strongly quasianalytic, there exists $g \in \mathcal{C}_{n+p}$ such that $\hat{g}=h$; hence, by quasianalyticity, $\phi(g)=f$; i.e. $g(x, \sigma(\lambda))=f(x, \lambda)$.

\section{Generic Division}

Lemma 3.1. Suppose that the system $\mathcal{C}$ has the property EH. Let $P\left(x_{n}, \lambda\right)$ be a generic polynomial in $x_{n}$ of degree $p$. If $f \in \mathcal{C}_{n}$, then there exist unique function germs $q \in \mathcal{C}_{n+p}$ and $r_{i} \in \mathcal{C}_{n-1+p}, 1 \leq i \leq p$, such that

$$
f(x)=P(x, \lambda) q(x, \lambda)+\sum_{i=1}^{p} r_{i}\left(x^{\prime}, \lambda\right) x_{n}^{p-i} .
$$

Proof. Set $x^{\prime}=\left(x_{1}, \ldots, x_{n-1}\right)$. We consider $f\left(x^{\prime}, x_{n}\right)$ and $f\left(x^{\prime}, \lambda_{1}\right)$ as elements of $\mathcal{C}_{n+p}$. Since $\mathcal{C}_{n+p}$ is closed under division by coordinates,

$$
f\left(x^{\prime}, x_{n}\right)-f\left(x^{\prime}, \lambda_{1}\right)=\left(x_{n}-\lambda_{1}\right) f_{1}(x, \lambda),
$$

with $f_{1} \in \mathcal{C}_{n+p}$ again. By repeating this process with $f_{1}$, we get:

$$
f_{1}\left(x^{\prime}, x_{n}, \lambda\right)-f_{1}\left(x^{\prime}, \lambda_{2}, \lambda\right)=\left(x_{n}-\lambda_{2}\right) f_{2}(x, \lambda),
$$

with $f_{2} \in \mathcal{C}_{n+p}$. At the end we get:

$$
f(x)=g(x, \lambda)\left(x_{n}-\lambda_{1}\right) \ldots\left(x_{n}-\lambda_{p}\right)+\sum_{i=1}^{p} g_{i}\left(x^{\prime}, \lambda\right) x_{n}^{p-i},
$$

with $g \in \mathcal{C}_{n+p}$ and $g_{i} \in \mathcal{A}_{R, n-1+p}, 1 \leq i \leq p$.

We see that $g$ and $g_{i}, 1 \leq i \leq p$, are symmetric with respect to $\lambda_{1}, \ldots, \lambda_{p}$. By Lemma 2.1, there exist $q \in \mathcal{C}_{n+p}$ and $r_{i} \in \mathcal{C}_{n-1+p}, 1 \leq i \leq p$, such that

$$
g=\phi(q), \quad g_{i}=\phi\left(r_{i}\right) \text {. }
$$

Then

$$
f=\phi(f)=\phi(q) \phi(P)+\sum_{i} \phi\left(r_{i}\right) x_{n}^{p-i}
$$


and this implies that

$$
f(x)=P(x, \lambda) q(x, \lambda)+\sum_{i=1}^{p} r_{i}\left(x^{\prime}, \lambda\right) x_{n}^{p-i},
$$

since $\phi$ is injective.

The uniqueness follows from the quasianalyticity of the system $\mathcal{C}$.

\section{WeIERSTRASS DIVISION THEOREM}

Let $f \in \mathcal{C}_{n}-\{0\}$. We say that $f$ is regular of order $p$ with respect to $x_{n}$ if the formal series $\hat{f}$ is regular of order $p$ with respect to $x_{n}$. For any $f \in \mathcal{C}_{n}-\{0\}$, after a linear invertible change of the coordinates $\left(x_{1}, \ldots, x_{n}\right)$, there is an integer $p \in \mathbb{N}$ such that $f$ is regular of order $p$ with respect to $x_{n}$.

Theorem 4.1. Suppose that the system $\mathcal{C}$ has the property EH. Let $f \in \mathcal{C}_{n}-\{0\}$ be regular of order $p$ with respect to $x_{n}$. Then for any $g \in \mathcal{C}_{n}$, there exist unique $q \in \mathcal{C}_{n}$ and $r_{i} \in \mathcal{C}_{n-1}, 1 \leq i \leq p$, such that

$$
g(x)=f(x) q(x)+\sum_{i=1}^{p} r_{i}\left(x^{\prime}\right) x_{n}^{p-i} .
$$

Proof. By Lemma 3.1, we can divide $f$ by the generic polynomial $P\left(x_{n}, \lambda\right)=x_{n}^{p}+$ $\sum_{i=1}^{p} \lambda_{i} x_{n}^{p-i}$. We have

$$
f(x)=P\left(x_{n}, \lambda\right) q(x, \lambda)+\sum_{i=1}^{p} r_{i}\left(x^{\prime}, \lambda\right) x_{n}^{p-i},
$$

with $q(x, \lambda) \in \mathcal{C}_{n+p}$ and $r_{i}\left(x^{\prime}, \lambda\right) \in \mathcal{C}_{n-1+p}, 1 \leq i \leq p$. Since $f$ is regular of order $p$ with respect $x_{n}$, we can easily see that

$$
q(0,0) \neq 0, \quad r_{i}(0,0)=0, \quad 1 \leq i \leq p,
$$

and the determinant of the matrix $\left(\frac{\partial r_{i}}{\partial \lambda_{j}}(0,0)\right)_{i, j=1 \ldots, p}$,

$$
\frac{D\left(r_{1}, \ldots, r_{p}\right)}{D\left(\lambda_{1}, \ldots, \lambda_{p}\right)}(0,0) \neq 0 .
$$

By the Implicit Function Theorem (condition $\left.\left(C_{4}\right)\right)$, there is $\psi\left(x^{\prime}\right)=\left(\psi_{1}\left(x^{\prime}\right) \ldots\right.$, $\left.\psi_{p}\left(x^{\prime}\right)\right) \in\left(\mathcal{C}_{n-1}\right)^{p}, \psi(0)=0$, such that $r_{i}\left(x^{\prime}, \psi\left(x^{\prime}\right)\right)=0,1 \leq i \leq p$. Hence

$$
f=q\left(x, \psi\left(x^{\prime}\right)\right) P\left(x^{\prime}, \psi\left(x^{\prime}\right)\right),
$$

since $q\left(x, \psi\left(x^{\prime}\right)\right)$ is invertible in $\mathcal{C}_{n}$. Then $f$ is equivalent in $\mathcal{C}_{n}$ to the polynomial

$$
x_{n}^{p}+\sum_{i=1}^{p} \psi_{i}\left(x^{\prime}\right) x_{n}^{p-i} .
$$

Now let $g \in \mathcal{C}_{n}$. We can divide $g$ by the generic polynomial $P\left(x_{n}, \lambda\right)$ and hence by $P\left(x_{n}, \psi\left(x^{\prime}\right)\right)$ after substituting $\lambda \rightarrow \psi\left(x^{\prime}\right)$. Since $q\left(x^{\prime}, \psi\left(x^{\prime}\right)\right)$ is invertible in $\mathcal{C}_{n}$, we have proved the existence. The uniqueness follows from the quasianalyticity of the system $\mathcal{C}$.

Corollary 4.1. We suppose that the system $\mathcal{C}=\left\{\mathcal{C}_{n}, n \in \mathbb{N}\right\}$ has the property $E H$. Then for each $n \in \mathbb{N}, \mathcal{C}_{n} \subseteq \mathbb{R}\left\{x_{1}, \ldots, x_{n}\right\}$.

Proof. We deduce Corollary 4.1 from Theorem 1.3 and Theorem 4.1 , 


\section{Appendix}

1) A way to yield differentiable quasianalytic systems is to consider the germs of smooth functions definable in a fixed polynomially bounded o-minimal structure which is an expansion of the ordered field of reals. For more details about an ominimal structure over the field of reals, we refer the reader to [6]. This system shares some good properties with the system of analytic rings. For instance, by [7, it is closed under composition, under differentiations, under division by coordinates, and under solutions of equations satisfying the hypothesis of the implicit function theorem. The Weierstrass division theorem never holds in this system (unless it is analytic) [4. Because of the lack of a Weierstrass division theorem, many problems remain open. For instance, these systems are not known to be Noetherian or to satisfy any kind of M. Artin-type of approximation theorem.

2) Let $\mathcal{E}(U)$ denote the ring of $C^{\infty}$ functions on an open set $U \subset \mathbb{R}^{n}$. As usual, for each $n$-tuple $\alpha=\left(\alpha_{1}, \ldots, \alpha_{n}\right) \in \mathbb{N}$, we set

$$
|\alpha|=\alpha_{1}+\ldots+\alpha_{n} \quad \text { and } \quad D^{\alpha}=\frac{\partial^{|\alpha|}}{\partial^{\alpha_{1}} \ldots \partial^{\alpha_{n}}} .
$$

Let $\rho>0$ and $M=\left\{M_{p}\right\}_{p=0}^{\infty}$ be an increasing sequence of positive real numbers. For each $f \in \mathcal{E}(U)$,

$$
\|f\|_{U, \rho, M}=\sup _{x \in U, p \in \mathbb{N}} \sup _{|\alpha|=p} \frac{\left|D^{\alpha} f(x)\right|}{\rho^{p} M_{p} p !}
$$

defines a norm on

$$
C_{M, \rho}(U)=\left\{f \in \mathcal{E}(U) /\|f\|_{U, \rho, M}<\infty\right\},
$$

which turns out to be a Banach space.

Definition 5.1. We say that $f \in \mathcal{E}(U)$ is in the Denjoy-Carleman class defined by $M\left(f \in C_{M}(U)\right.$ for short) if any point $x \in U$ admits a neighborhood $U_{x} \subset U$ such that $f \in C_{M, \rho}\left(U_{x}\right)$ for some $\rho$.

Denote by the cube in $\mathbb{R}^{n}, \Delta_{n}(r)=\left\{\left(x_{1}, \ldots, x_{n}\right) \in \mathbb{R}^{n} /\left|x_{i}\right|<r\right.$ for $\left.1 \leq i \leq n\right\}$. Let $C_{M}\left(\mathbb{R}^{n}, 0\right)$ be the inductive limit of $C_{M}\left(\Delta_{n}(r)\right)$ when $r \rightarrow \infty$.

In order to get classes of functions with some good structural properties, we require two conditions on the sequence $M=\left\{M_{p}\right\}_{p=0}^{\infty}$; see [3]:

$$
\begin{gathered}
M=\left\{M_{p}\right\}_{p=0}^{\infty} \quad \text { is logarithmically convex, and } M_{0}=1, \\
\sup _{p}\left(\frac{M_{p+1}}{M_{p}}\right)^{\frac{1}{p}}<\infty .
\end{gathered}
$$

Proposition 5.2 (3) $) \cdot C_{M}\left(\mathbb{R}^{n}, 0\right)$ is a local ring containing $\mathbb{R}\left\{x_{1}, \ldots, x_{n}\right\}$ with maximal ideal; $m=\left\{f \in C_{M}\left(\mathbb{R}^{n}, 0\right) / f(0)=0\right\}=\left(x_{1}, \ldots, x_{n}\right) C_{M}\left(\mathbb{R}^{n}, 0\right)$.

- $C_{M}\left(\mathbb{R}^{n}, 0\right)$ is closed under composition: If $f \in C_{M}\left(\mathbb{R}^{n}, 0\right)$ and $g=\left(g_{1}, \ldots, g_{n}\right) \in$ $\left[C_{M}\left(\mathbb{R}^{k}, 0\right)\right]^{n}$ with $g(0)=0$, then $f \circ g=f\left(g_{1}, \ldots, g_{n}\right) \in C_{M}\left(\mathbb{R}^{k}, 0\right)$.

- $C_{M}\left(\mathbb{R}^{n}, 0\right)$ is closed under differentiation: If $f \in C_{M}\left(\mathbb{R}^{n}, 0\right)$, then $\frac{\partial f}{\partial x_{j}} \in$ $C_{M}\left(\mathbb{R}^{n}, 0\right), j=1, \ldots, n$.

- $C_{M}\left(\mathbb{R}^{n}, 0\right)$ is closed under division by coordinates: If $f \in C_{M}\left(\mathbb{R}^{n}, 0\right)$ and $f\left(x_{1}, \ldots, x_{i-1}, 0, x_{i+1}, \ldots x_{n}\right)$ is identically zero, then $f(x)=x_{i} g(x)$, with $g \in$ $C_{M}\left(\mathbb{R}^{n}, 0\right)$.

- The Implicit Function Theorem holds for $C_{M}\left(\mathbb{R}^{n}, 0\right)$ (in particular these rings are henselian rings): Let $f=\left(f_{1}, \ldots, f_{m}\right) \in\left[C_{M}\left(\mathbb{R}^{n+m}, 0\right)\right]^{m}$, with $f(0,0)=0$ and 
$\operatorname{det}\left[\frac{\partial f_{i}}{\partial y_{j}}\right]_{i, j=1, \ldots, m}(0,0) \neq 0$. Then, there is a (unique) $g=\left(g_{1}, \ldots, g_{m}\right) \in C_{M}\left(\mathbb{R}^{n}, 0\right)$ with $g(0)=0$ such that $f(g(x)=0$.

In order to deal with quasianalytic classes we also require the following condition:

$$
\sum_{p} \frac{M_{p}}{(p+1) M_{p+1}}=\infty
$$

Hadamard raised the question of characterizing the sequences $M$ that yield quasianalytic classes. The Denjoy-Carleman Theorem is an answer to this problem [3, 8 .

Theorem 5.3 (Denjoy-Carleman Theorem). Let $U \subset \mathbb{R}^{n}$ be an open set and let $M$ be a sequence of positive real numbers satisfying condition 5.1. The following conditions are equivalent:

a. $C_{M}(U)$ is quasianalytic,

b. $C_{M}(U)$ does not contain functions with compact support,

c. $M$ satisfies condition 5.3 .

\section{REFERENCES}

[1] S.S. Abhyankar and M. van der Put. Homomorphisms of analytic local rings. J. Reine Angew. Math. 242 (1970), 26-60. MR.0260729 (41:5353)

[2] P.M. Eakin and G.A. Harris. When $F(f)$ convergent implies $f$ is convergent. Math. Ann. 229 (1977), 201-210. MR0444651 (56:3001)

[3] C.L. Childress. Weierstrass division in quasianalytic local rings. Canad. J. Math. 28 (5) (1975), 938-953. MR 0417441 (54:5491)

[4] A. Elkhadiri and H. Sfouli. Weierstrass division theorem in quasianalytic local rings. Studia Mathematica 185 (1) (2008), 83-86. MR2380000

[5] H. Komatsu. The implicit function theorem of ultradifferentiable mappings. Proc. Japan Acad. Ser. A Math. Sci. 55 (1979), 69-72. MR531445 (80e:58007)

[6] L. van den Dries. Tame topology and o-minimal structures. London Mathematical Society Lecture Note Series, 248. Cambridge University Press, Cambridge, 1998. MR1633348 (99j:03001)

[7] C. Miller. Infinite differentiability in polynomially bounded o-minimal structures. Proc. Amer. Math. Soc. 123 (1995), 2551-2555. MR1257118 (95j:03069)

[8] W. Rudin. Real and complex analysis. McGraw-Hill, New York, 1966. MR0210528 (35:1420)

Department of Mathematics, Faculty of Sciences, University Ibn Tofail, BP 133 KÉNITRA, MoROCCO

E-mail address: kabdelhafed@hotmail.com 